\title{
Solution Intervals for Variables in Spatial RCRCR Linkages
}

\author{
Enric Celaya \\ Institut de Robòtica i Informàtica Industrial (CSIC-UPC) \\ Llorens Artigas 4-6, 08028 Barcelona, Spain \\ E-mail: celaya@iri.upc.edu
}

\begin{abstract}
An analytic method to compute the solution intervals for the input variables of spatial RCRCR linkages and their inversions is presented. The input-output equation is formulated as the intersection of a single ellipse with a parameterized family of ellipses, both related with the possible values that certain dual angles determined by the configuration of the mechanism can take. Bounds for the angles of the input pairs of the RCRCR and RRCRC inversions are found by imposing the tangency of two ellipses, what reduces to analyzing the discriminant of a fourth degree polynomial. The bounds for the input pair of the RCRRC inversion is found as the intersection of a single ellipse with the envelope of the parameterized family of ellipses. The method provides the bounds of each of the assembly modes of the mechanism as well as the local extrema that may exist for the input variable.
\end{abstract}

Keywords: Solution intervals, 3R2C linkages, position analysis, forward and inverse kinematics.

\section{Introduction}

A core task of Kinematics is that of finding the input-output relationships for mechanisms of interest. While an input-output equation contains all the essential information relating the input and output variables, some important aspects relevant for the analysis and synthesis of a mechanism are only implicit in an input-output equation. Thus, for example, an input-output equation does not make explicit whether a mechanism with given link dimensions can actually be assembled or not, nor if the mechanism can be assembled in one or more different ways, nor what is the mobility range of a particular mode of assembly. Such kind of information is directly available when the solution intervals of the input variables are given explicitly.

The determination of the range of motion of a joint is a classical subject in planar linkages, where the Grashof rules for planar quadrilaterals are commonly used to decide if a given joint can perform full rotations and to determine the extreme positions of the non fully rotatable joints. A recent work addressing the determination of the feasible ranges for joints in planar four-bar linkages is [1]. A more comprehensive study of the solution intervals for variables in planar and spherical linkages can be found in [2], where the problem is solved in general for arbitrary single-loop linkages with any number of links joined by $\mathrm{R}$ and $\mathrm{P}$ pairs. Through the use of the spherical indicatrix, the method has been extended to find the solution intervals of any spatial single-loop mechanism having at least three translational d.o.f., as well as of a certain class of spatial mechanisms called triangulable [3]. Further extensions allowed to obtain the solution intervals for multiple-loop planar and spherical linkages via interval propagation algorithms $[4,5]$. The contribution of the present work is that of obtaining in an analytic way the solution intervals for a spatial linkage not previously dealt with.

In principle, solution intervals for a given variable can be approximated by repeatedly solving the input-output equation for the desired variable: depending on whether there are real solutions or not for a given value of the input variable, it must be included or excluded from a solution interval. Many earlier works on kinematic analysis of mechanisms used to make a rough estimation of the solution intervals by sampling the input variable with a given discretization to plot the values obtained for the output variable, and then infer the valid intervals from the figure. For example, in the analysis of the same RCRCR mechanism used here, in [6] we can read: "we may observe from the four output curves that there is no solution of [the output angle] within the range $10^{\circ}<$ input angle] $<80^{\circ}$ ", what is a very rough approximation of the correct interval (corresponding to $\theta_{5}$ in Table 2). 
More accurate approximations can be obtained by progressively increasing the sampling resolution, as in [7], that, after plotting the input-output function by initially sampling the input variable by steps of $1^{\circ}$, the interval bounds are refined until the third decimal position to give the values $69.350^{\circ}<\theta_{1}<410.471^{\circ}$ for one assembly mode, and $148.788^{\circ}<\theta_{1}<308.299^{\circ}$ for the second, in full accordance with the results presented here (see Table 2). The limitation of this approach is that it can only find the bounding values within the precision of the discretization used initially and, in the extreme case of a mechanism with isolated solutions or very short solution intervals, they may be completely missed.

To avoid the potential loss of solutions, numerical approaches have been developed using iterative branch-andprune methods to approximate the solution set by a collection of covering boxes defined in the configuration space of the linkage with a resolution specified by the user [8,9]. Given such a box covering, approximate solution intervals can be obtained by projection on the desired variable axis. While this approach grants that no solution is excluded from the approximation, it works iteratively, and can only provide intervals enclosing the solution with a predefined precision. Observe that the aim of this approach is different from that of computing intervals guaranteed to contain the actual value taken by the output variable, which is the goal of interval analysis methods [10]. The goal is, instead, to accurately determine the range of values that a variable can take to give rise to a feasible configuration.

From a theoretical point of view, the limit positions of a joint correspond to stationary configurations of the linkage. In [11], a condition for the existence of a stationary configuration is given in terms of the screw system theory. In [12], this condition is used to derive an extra relationship to determine a limit position for a desired joint variable in general spatial mechanisms and, in [13], an alternative formulation is presented using the reciprocal screw system. The method is illustrated with a five-bar RCCRR, but the author refuses to compute numerical solutions due to its complexity, which would require the simultaneous solution of a system of five equations involving products of sine and cosine of five unknown angles. A more tractable approach is presented in [14], which uses polynomial discriminants to bound the solution intervals, but the procedure is only applied to 4-bar mechanisms.

A completely different approach is that of [15], which applies the Morse-Bott theory to determine the maximum and minimum reach of revolute-jointed manipulators. The interval of possible distances reachable by the manipulator can be seen as the feasible interval for the length of an extra link connecting the base to the end-point. Unfortunately, this strategy is not directly applicable to get the solution range for variables of most linkages.

Nowadays, a general and computationally effective procedure to find the solution intervals of arbitrary spatial mechanisms is still lacking. In the present work we make a further step in this direction by solving the case of the spatial RCRCR mechanism and its inversions, namely, RCRRC and RRCRC.

The paper is organized as follows: Sections 2 and 3 are a short presentation of the concepts of dual numbers and their application to kinematics, while Sections 4 and 5 reformulate known results for the derivation of the input-output functions of the RCRCR mechanism. The novel results are presented in Section 6, where the the bounding values for the input variables of the mechanism are found analytically, and Section 7 where the solution intervals are determined. Finally, Section 8 closes the paper with some conclusions.

\section{Formulation of kinematic equations with dual numbers}

A dual number $\hat{x}$ is defined as the sum of a real and a dual component $\hat{x}=x+\varepsilon x_{0}$. The dual component is a multiple of the dual unit $\varepsilon$, which by definition has the property $\varepsilon^{2}=0$. The sum and product of two dual numbers are given by:

$$
\begin{aligned}
& \hat{x}+\hat{y}=\left(x+\varepsilon x_{0}\right)+\left(y+\varepsilon y_{0}\right)=(x+y)+\varepsilon\left(x_{0}+y_{0}\right) \\
& \hat{x} \hat{y}=\left(x+\varepsilon x_{0}\right)\left(y+\varepsilon y_{0}\right)=x y+\varepsilon\left(x y_{0}+y x_{0}\right)
\end{aligned}
$$

In general, a function of a dual variable can be obtained from its Taylor's series expansion, which using $\varepsilon^{n}=0,(n \geq 2)$, gives:

$$
f\left(x+\varepsilon x_{0}\right)=f(x)+\varepsilon x_{0} \frac{d f}{d x}(x)
$$

In particular, the trigonometric functions sin and cos of a dual variable are:

$$
\begin{aligned}
& \sin \hat{x}=\sin x+\varepsilon x_{0} \cos x \\
& \cos \hat{x}=\cos x-\varepsilon x_{0} \sin x
\end{aligned}
$$


All trigonometric identities valid for real variables are also valid for dual variables.

In the same way that a real value can be used to represent the angle between two vectors in space, a dual number can be used to represent the angle and perpendicular distance between two lines in space. Thus, the twist angle $\alpha_{i j}$ and link length $a_{i j}$ describing the fixed parameters of a link can be represented by the dual angle $\hat{\alpha}_{i j}=\alpha_{i j}+\varepsilon a_{i j}$. Similarly, the variable parameters of a $\mathrm{C}$ pair can be represented by the dual angle $\hat{\theta}_{i}=\theta_{i}+\varepsilon t_{i}$, where $\theta_{i}$ is the angular displacement and $t_{i}$ the joint offset. In the case of a $\mathrm{R}$ pair, the same representation is used with the difference that in this case $t_{i}$ is fixed. A dual rotation of angle $\hat{\alpha}=\alpha+\varepsilon a$ and axis $\boldsymbol{k}$ is defined as the composition of a real rotation of angle $\alpha$ around axis $\boldsymbol{k}$, and a translation of length $a$ along the same axis.

A spatial transformation involving a translation of vector $\boldsymbol{v}=\left[v_{x}, v_{y}, v_{z}\right]^{\top}$ and a rotation $\mathbf{R}$ can be represented by a dual-number rotation matrix $\hat{\mathbf{R}}=\mathbf{R}+\varepsilon \mathbf{D}$, where the real component $\mathbf{R}$ is an orthogonal matrix corresponding to the rotation part, and the dual component is $\mathbf{D}=\mathbf{P}_{v} \mathbf{R}$, where $\mathbf{P}_{v}$ is a skew-symmetric matrix obtained from the coordinates of $\boldsymbol{v}$ as [16]:

$$
\mathbf{P}_{v}=\left(\begin{array}{rrr}
0 & -v_{z} & v_{y} \\
v_{z} & 0 & -v_{x} \\
-v_{y} & v_{x} & 0
\end{array}\right)
$$

In particular, dual rotations about the $x$ and $z$ axes, describing a pair of dual angle $\hat{\theta}_{i}$ and a link of dual angle $\hat{\alpha}_{i j}$, respectively, can be written as:

$$
\hat{\mathbf{R}} \mathbf{x}\left(\hat{\theta}_{i}\right)=\left(\begin{array}{rrr}
1 & 0 & 0 \\
0 & \cos \hat{\theta}_{i} & -\sin \hat{\theta}_{i} \\
0 & \sin \hat{\theta}_{i} & \cos \hat{\theta}_{i}
\end{array}\right), \quad \hat{\mathbf{R}} \mathbf{z}\left(\hat{\alpha}_{i j}\right)=\left(\begin{array}{rrr}
\cos \hat{\alpha}_{i j} & -\sin \hat{\alpha}_{i j} & 0 \\
\sin \hat{\alpha}_{i j} & \cos \hat{\alpha}_{i j} & 0 \\
0 & 0 & 1
\end{array}\right) .
$$

Thanks to the principle of transference of Kotelnikov, the loop equation of a spatial mechanism can be formulated with dual-number matrices in the same way as its corresponding spherical mechanism is formulated with real matrices.

\section{Dual Euler's decomposition of a spatial transformation}

The Euler's decomposition allows expressing any 3D rotation as the product of three rotations about the $x-z-x$ axes:

$$
\mathbf{R}=\mathbf{R} \mathbf{x}(\varphi) \mathbf{R z}(\phi) \mathbf{R x}(\psi) .
$$

This decomposition is not unique since $\mathbf{R x}(\varphi) \mathbf{R z}(\phi) \mathbf{R} \mathbf{x}(\psi)=\mathbf{R} \mathbf{x}(\varphi+\pi) \mathbf{R z}(-\phi) \mathbf{R} \mathbf{x}(\psi+\pi)$, however, we can impose uniqueness by choosing $\phi \in[0, \pi)$. Similarly, we can also express any spatial transformation as a product of three dual angle rotations along the $x-z-x$ axes. Thus, a spatial transformation involving a translation $v=\left[v_{x}, v_{y}, v_{z}\right]^{\top}$ and a rotation $\mathbf{R}$ can be represented by:

$$
\hat{\mathbf{R}}=\hat{\mathbf{R}} \mathbf{x}(\hat{\varphi}) \hat{\mathbf{R}} \mathbf{z}(\hat{\phi}) \hat{\mathbf{R}} \mathbf{x}(\hat{\psi})=\mathbf{R}+\varepsilon \mathbf{D},
$$

where $\hat{\varphi}=\varphi+\varepsilon p, \hat{\phi}=\phi+\varepsilon q, \hat{\psi}=\psi+\varepsilon r$. The dual components $p, q, r$ may be obtained from the relation $\mathbf{P}_{v}=\mathbf{D} \mathbf{R}^{-1}$ and using (1) to get the system of equations:

$$
\left\{\begin{array}{l}
v_{x}=r * \cos \phi+p \\
v_{y}=r * \cos \varphi \sin \phi-q \sin \varphi \\
v_{z}=r * \sin \varphi \sin \phi+q \cos \varphi
\end{array}\right.
$$

Solving for $p, q, r$ we get:

$$
\begin{aligned}
p & =v_{x}-\left(v_{y} \cos \varphi \cos \phi+v_{z} \sin \varphi \cos \phi\right) / \sin \phi \\
q & =-v_{y} \sin \varphi+v_{z} \cos \varphi \\
r & =\left(v_{y} \cos \varphi+v_{z} \sin \varphi\right) / \sin \phi .
\end{aligned}
$$

Note that this solution is not valid when $\phi=0$. In such case $\mathbf{R}$ reduces to a rotation around $x: \mathbf{R x}(\xi)=\mathbf{R x}(\varphi+\psi)$, and the angles $\varphi$ and $\psi$ are not uniquely determined by the usual Euler's decomposition. In this case, the system (4) becomes:

$$
\left\{\begin{array}{l}
v_{x}=r+p \\
v_{y}=-q \sin \varphi \\
v_{z}=q \cos \varphi
\end{array}\right.
$$


According to the first equation, we can take $p$ as a free parameter, and $r=v_{x}-p$. In this case, the values of $q$ and $\varphi$ are obtained by solving the system of the last two equations as:

$$
\begin{aligned}
q & =\sqrt{v_{y}^{2}+v_{z}^{2}} \\
\varphi & =\arctan \left(-v_{y}, v_{z}\right)
\end{aligned}
$$

and finally, $\psi$ is determined as $\psi=\xi-\varphi$.

\section{Loop equations for the RCRCR mechanism}

The RCRCR mechanism is described by the loop equation:

$(\mathrm{R})$

(C)

$(\mathrm{R})$

$$
\mathbf{I}=\hat{\mathbf{R}} \mathbf{x}\left(\hat{\theta}_{1}\right) \hat{\mathbf{R}} \mathbf{z}\left(\hat{\alpha}_{12}\right) \hat{\mathbf{R}} \mathbf{x}\left(\hat{\theta}_{2}\right) \hat{\mathbf{R}} \mathbf{z}\left(\hat{\alpha}_{23}\right) \hat{\mathbf{R}} \mathbf{x}\left(\hat{\theta}_{3}\right) \hat{\mathbf{R}} \mathbf{z}\left(\hat{\alpha}_{34}\right) \hat{\mathbf{R}} \mathbf{x}\left(\hat{\theta}_{4}\right) \hat{\mathbf{R}} \mathbf{z}\left(\hat{\alpha}_{45}\right) \hat{\mathbf{R}} \mathbf{x}\left(\hat{\theta}_{5}\right) \hat{\mathbf{R}} \mathbf{z}\left(\hat{\alpha}_{51}\right)
$$

where $\hat{\alpha}_{i j}=\alpha_{i j}+\varepsilon a_{i j}$ and $\hat{\theta}_{i}=\theta_{i}+\varepsilon t_{i}$ are dual angles corresponding to the link dimensions and joint variables, respectively, and an $(\mathrm{R})$ or $(\mathrm{C})$ above each $\hat{\mathbf{R}} \mathbf{x}$ operator indicates if the pair is rotational or cylindric, respectively. We rearrange (11) so as to have a $\mathrm{C}$ pair at both ends of the right hand side:

$$
(\mathrm{R}) \quad(\mathrm{C}) \quad(\mathrm{R}) \quad(\mathrm{R}) \quad(\mathrm{C})
$$

$$
\hat{\mathbf{R}} \mathbf{z}\left(-\hat{\alpha}_{34}\right) \hat{\mathbf{R}} \mathbf{x}\left(-\hat{\theta}_{3}\right) \hat{\mathbf{R}} \mathbf{z}\left(-\hat{\alpha}_{23}\right)=\hat{\mathbf{R}} \mathbf{x}\left(\hat{\theta}_{4}\right) \hat{\mathbf{R}} \mathbf{z}\left(\hat{\alpha}_{45}\right) \hat{\mathbf{R}} \mathbf{x}\left(\hat{\theta}_{5}\right) \hat{\mathbf{R}} \mathbf{z}\left(\hat{\alpha}_{51}\right) \hat{\mathbf{R}} \mathbf{x}\left(\hat{\theta}_{1}\right) \hat{\mathbf{R}} \mathbf{z}\left(\hat{\alpha}_{12}\right) \hat{\mathbf{R}} \mathbf{x}\left(\hat{\theta}_{2}\right) .
$$

Applying the dual Euler's decomposition to both sides, excluding the cylindric pairs of $\hat{\theta}_{4}$ and $\hat{\theta}_{2}$, we write:

$$
\begin{aligned}
\hat{\mathbf{R}} \mathbf{z}\left(-\hat{\alpha}_{34}\right) \hat{\mathbf{R}} \mathbf{x}\left(-\hat{\theta}_{3}\right) \hat{\mathbf{R}} \mathbf{z}\left(-\hat{\alpha}_{23}\right) & =\hat{\mathbf{R}} \mathbf{x}\left(\hat{\varphi}_{1}\right) \hat{\mathbf{R}} \mathbf{z}\left(\hat{\phi}_{1}\right) \hat{\mathbf{R}} \mathbf{x}\left(\hat{\psi}_{1}\right) \\
\hat{\mathbf{R}} \mathbf{z}\left(\hat{\alpha}_{45}\right) \hat{\mathbf{R}} \mathbf{\mathbf { R }}\left(\hat{\theta}_{5}\right) \hat{\mathbf{R}} \mathbf{z}\left(\hat{\alpha}_{51}\right) \hat{\mathbf{R}} \mathbf{x}\left(\hat{\theta}_{1}\right) \hat{\mathbf{R}} \mathbf{z}\left(\hat{\alpha}_{12}\right) & =\hat{\mathbf{R}} \mathbf{x}\left(\hat{\varphi}_{2}\right) \hat{\mathbf{R}} \mathbf{z}\left(\hat{\phi}_{2}\right) \hat{\mathbf{R}} \mathbf{x}\left(\hat{\psi}_{2}\right)
\end{aligned}
$$

and substituting in equation (12):

$$
\hat{\mathbf{R}} \mathbf{x}\left(\hat{\varphi}_{1}\right) \hat{\mathbf{R}} \mathbf{z}\left(\hat{\phi}_{1}\right) \hat{\mathbf{R}} \mathbf{x}\left(\hat{\psi}_{1}\right)=\hat{\mathbf{R}} \mathbf{x}\left(\hat{\theta}_{4}+\hat{\varphi}_{2}\right) \hat{\mathbf{R}} \mathbf{z}\left(\hat{\phi}_{2}\right) \hat{\mathbf{R}} \mathbf{x}\left(\hat{\psi}_{2}+\hat{\theta}_{2}\right) .
$$

Due to the uniqueness of the Euler's decomposition for $\phi \in[0, \pi)$, a necessary condition for (15) to be fulfilled is $\hat{\phi}_{1}= \pm \hat{\phi}_{2}$, which can be expressed equivalently as $\cos \hat{\phi}_{1}=\cos \hat{\phi}_{2}$. Note that this condition is also sufficient since $\hat{\theta}_{4}$ and $\hat{\theta}_{2}$ correspond to $C$ pairs, so that their real and dual parts can be chosen to satisfy $\hat{\theta}_{4}+\hat{\varphi}_{2}=\hat{\varphi}_{1}$ and $\hat{\psi}_{2}+\hat{\theta}_{2}=\hat{\psi}_{1}$. The value of $\cos \hat{\phi}_{1}$ only depends on the rotational variable $\theta_{3}$, and is given by the $(1,1)$ matrix element of the left hand side of (13). Writing $\hat{\phi}_{1}=\phi_{1}+\varepsilon d_{1}$, the real and dual parts of $\cos \hat{\phi}_{1}$ as functions of $\theta_{3}$ are given by:

$$
\left\{\begin{array}{l}
\cos \phi_{1}=A+B \cos \theta_{3} \\
d_{1} \sin \phi_{1}=C+D \cos \theta_{3}+E \sin \theta_{3}
\end{array}\right.
$$

with

$$
\begin{aligned}
& A=c_{34} c_{23} \\
& B=-s_{34} s_{23} \\
& C=-a_{23} s_{23} c_{34}-a_{34} s_{34} c_{23} \\
& D=-a_{34} s_{23} c_{34}-a_{23} s_{34} c_{23} \\
& E=t_{3} s_{34} s_{23}
\end{aligned}
$$

where $c_{i j}=\cos \alpha_{i j}$ and $s_{i j}=\sin \alpha_{i j}$.

By its side, the value of $\cos \hat{\phi}_{2}$ depends on the rotational variables $\theta_{1}$ and $\theta_{5}$, and is given by the $(1,1)$ matrix element of the left hand side of (14). Writing $\hat{\phi}_{2}=\phi_{2}+\varepsilon d_{2}$, the real and dual parts of $\cos \hat{\phi}_{2}$ as functions of $\theta_{1}$ and $\theta_{5}$ are given by:

$$
\left\{\begin{array}{l}
\cos \phi_{2}=F+G \cos \theta_{1}+H \sin \theta_{1} \\
d_{2} \sin \phi_{2}=J+K \cos \theta_{1}+L \sin \theta_{1}
\end{array}\right.
$$

where $F, G, H, J, K, L$ are functions of $\theta_{5}$ : 


$$
\begin{aligned}
& F=c_{12} c_{45} c_{51}-c_{12} s_{45} s_{51} \cos \theta_{5} \\
& G=-s_{12} c_{45} s_{51}-s_{12} s_{45} c_{51} \cos \theta_{5} \\
& H=s_{45} s_{12} \sin \theta_{5} \\
& J=s_{45} s_{51} t_{5} c_{12} \sin \theta_{5}+\left(s_{45} s_{51} a_{12} s_{12}-\left(a_{45} c_{45} s_{51}+s_{45} c_{51} a_{51}\right) c_{12}\right) \cos \theta_{5}-c_{45} c_{51} a_{12} s_{12}-\left(c_{45} a_{51} s_{51}+a_{45} s_{45} c_{51}\right) c_{12} \\
& K=\left(c_{51} t_{5}+t_{1}\right) s_{45} s_{12} \sin \theta_{5}-\left(c_{51} s_{45} a_{12} c_{12}+\left(a_{45} c_{45} c_{51}-s_{45} s_{51} a_{51}\right) s_{12}\right) \cos \theta_{5}-c_{45} s_{51} a_{12} c_{12}-\left(c_{45} a_{51} c_{51}-a_{45} s_{45} s_{51}\right) s_{12} \\
& L=\left(c_{45} a_{45} s_{12}+s_{45} a_{12} c_{12}\right) \sin \theta_{5}+\left(c_{51} t_{1}+t_{5}\right) s_{45} s_{12} \cos \theta_{5}+c_{45} s_{51} s_{12} t_{1}
\end{aligned}
$$

In (17), we collected the terms on $\theta_{1}$ to visualize the linear dependency of $\hat{\phi}_{2}$ on $\sin \theta_{1}$ and $\cos \theta_{1}$. Clearly, the dependence of $\hat{\phi}_{2}$ on $\sin \theta_{5}$ and $\cos \theta_{5}$ is also linear, since variables $\theta_{1}$ and $\theta_{5}$ play equivalent roles. Using (16) and (17), the condition $\cos \hat{\phi}_{1}=\cos \hat{\phi}_{2}$ gives rise to the system:

$$
\begin{cases}A+B \cos \theta_{3} & =F+G \cos \theta_{1}+H \sin \theta_{1} \\ C+D \cos \theta_{3}+E \sin \theta_{3} & =J+K \cos \theta_{1}+L \sin \theta_{1}\end{cases}
$$

Summarizing, equations (18) are the necessary and sufficient condition for the RCRCR mechanism to close, and only involve the real variables of the rotational pairs $\theta_{1}, \theta_{3}$, and $\theta_{5}$.

\section{Input-output functions}

We consider the three inversions of the mechanism where the input is the angular variable of a rotational pair: RCRCR, RRCRC, and RCRRC. For each inversion, the angle of the first R pair is assumed to be the input variable and two input-output functions are considered, one for each of the other two R pairs. All inversions involve the same loop equation (18) and, to obtain the input-output function between an input and an output variable, we only need to eliminate the third variable from the equations. Thus, in what follows, we will refer to the input-output function $\theta_{j}\left(\theta_{i}\right)$ of an RCRCR mechanism, irrespective of the involved inversion, whichever are the input $\theta_{i}$ and output $\theta_{j}$.

\subsection{Input-output function $\theta_{1}\left(\theta_{5}\right)$}

The input-output function $\theta_{1}\left(\theta_{5}\right)$ can be obtained by eliminating $\theta_{3}$ from (18). This can be done by isolating $\cos \theta_{3}$ from the first equation, and $\sin \theta_{3}$ from the equation obtained with the appropriate combination of the two equations to eliminate $\cos \theta_{3}$, and then using the identity $\sin ^{2} \theta_{3}+\cos ^{2} \theta_{3}=1$. The result is a single equation involving a 2 nd degree polynomial in $\sin \theta_{1}$ and $\cos \theta_{1}$ whose coefficients are in turn 2 nd degree polynomials of $\sin \theta_{5}$ and $\cos \theta_{5}$. Applying the tangent half-angle substitution $T_{1}=\tan \left(\theta_{1} / 2\right)$, we obtain a fourth-degree polynomial for $T_{1}$ whose coefficients depend on $\theta_{5}$. For each given value of the input angle $\theta_{5}$, the corresponding value of $\theta_{1}$ can be obtained computing the roots of the resulting fourth-degree polynomial.

\subsection{Input-output function $\theta_{3}\left(\theta_{5}\right)$}

Once $\theta_{1}$ has been obtained for a given value of the input angle $\theta_{5}$, the corresponding value for $\theta_{3}$ is readily obtained by substituting the values of $\theta_{5}$ and $\theta_{1}$ in the previously isolated expressions for $\sin \theta_{3}$ and $\cos \theta_{3}$, from which $\theta_{3}$ is determined. Alternatively, $\theta_{3}\left(\theta_{5}\right)$ could be directly obtained by eliminating $\theta_{1}$ instead of $\theta_{3}$ in (18) and using a similar procedure to get a fourth-degree polynomial for $T_{3}=\tan \left(\theta_{3} / 2\right)$ though, in this case, the coefficients of $T_{3}$ involve 4 th degree polynomials of $\sin \theta_{5}$ and $\cos \theta_{5}$, making the computation slightly less efficient.

\subsection{Input-output function $\theta_{5}\left(\theta_{3}\right)$}

To obtain the input-output function $\theta_{5}\left(\theta_{3}\right)$ we must eliminate $\theta_{1}$ from (18), but in this case, the elimination process gives rise to expressions for $\sin \theta_{1}$ and $\cos \theta_{1}$ that are quadratic in $\sin \theta_{5}$ and $\cos \theta_{5}$, and the identity $\sin ^{2} \theta_{1}+\cos ^{2} \theta_{1}=1$ involves a fourth-degree polynomial in $\sin \theta_{5}$ and $\cos \theta_{5}$, so that computing $\theta_{5}\left(\theta_{3}\right)$ requires finding the roots of an 8th degree polynomial in $T_{5}=\tan \left(\theta_{5} / 2\right)$ for each input value $\theta_{3}$, what can be done numerically. 


\subsection{Input-output functions $\theta_{5}\left(\theta_{1}\right), \theta_{3}\left(\theta_{1}\right)$ and $\theta_{1}\left(\theta_{3}\right)$}

By interchanging the roles of $\theta_{1}$ and $\theta_{5}$ in the above derivations, completely analogous procedures can be applied to obtain the input-output functions $\theta_{5}\left(\theta_{1}\right), \theta_{3}\left(\theta_{1}\right)$ and $\theta_{1}\left(\theta_{3}\right)$.

Figure 1 shows the different input-output functions obtained by regular sampling of the input variable for a mechanism with link dimensions given in Table 1, in coincidence with those shown in [17] and [18]. Only the input-output functions $\theta_{1}\left(\theta_{5}\right), \theta_{3}\left(\theta_{5}\right)$ and $\theta_{1}\left(\theta_{3}\right)$ are shown since the other three, namely $\theta_{1}\left(\theta_{5}\right), \theta_{5}\left(\theta_{3}\right)$ and $\theta_{3}\left(\theta_{1}\right)$, are respectively identical to them after interchanging the vertical and horizontal axes.

Table 1: Parameters of the links of the example RCRCR mechanism

\begin{tabular}{|c|c|c|c|c|}
\hline$\alpha_{12}=60^{\circ}$ & $\alpha_{23}=45^{\circ}$ & $\alpha_{34}=35^{\circ}$ & $\alpha_{45}=30^{\circ}$ & $\alpha_{51}=10^{\circ}$ \\
\hline$a_{12}=25$ & $a_{23}=30$ & $a_{34}=40$ & $a_{45}=10$ & $a_{51}=32$ \\
\hline$t_{1}=30$ & & $t_{3}=25$ & & $t_{5}=0$ \\
\hline
\end{tabular}
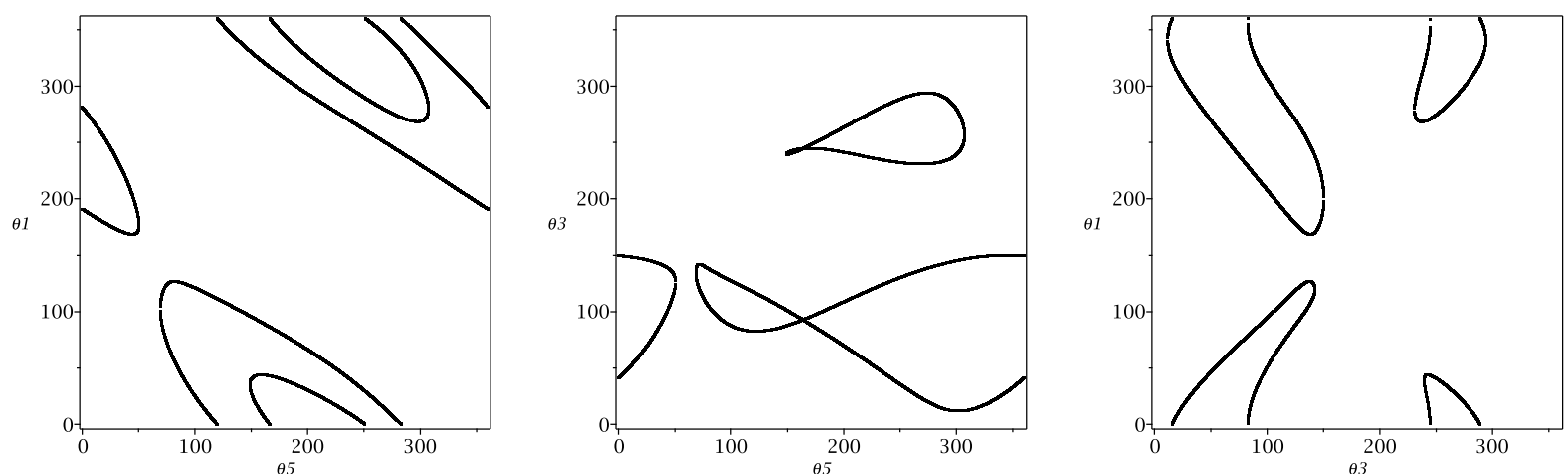

Figure 1: Input-output functions $\theta_{1}\left(\theta_{5}\right), \theta_{3}\left(\theta_{5}\right)$, and $\theta_{1}\left(\theta_{3}\right)$ for the RCRCR mechanism with the link dimensions of Table 1

\section{Determination of the bounding values of the input variables}

Equations (16) are the parametric equations of an ellipse in the plane with coordinates $(x, y)=\left(\cos \phi_{1}, d_{1} \sin \phi_{1}\right)$, and represent the possible values that the dual number $\cos \hat{\phi}_{1}\left(\theta_{3}\right)$ may take when $\theta_{3}$ takes values in $[0,2 \pi]$. Similarly, equations (17) can be seen as a parameterized family (with parameter $\theta_{5}$ ) of parametric equations of ellipses (with parameter $\left.\theta_{1}\right)$ in the plane with coordinates $(x, y)=\left(\cos \phi_{2}, d_{2} \sin \phi_{2}\right)$, and represent the possible values that the dual number $\cos \hat{\phi}_{2}\left(\theta_{1}, \theta_{5}\right)$ may take when $\theta_{1}$ and $\theta_{5}$ take values in $[0,2 \pi]$. In Figure 2, left, the ellipse of the possible values of $\cos \hat{\phi}_{1}\left(\theta_{3}\right)$ is represented in blue, and a number of ellipses with different values of parameter $\theta_{5}$ in the family of ellipses $\cos \hat{\phi}_{2}\left(\theta_{1}, \theta_{5}\right)$ are represented in red for the link parameters of Table 1. Alternatively, equations (17) can also be seen as a parameterized family (with parameter $\theta_{1}$ ) of parametric equations of ellipses (with parameter $\theta_{5}$ ), as represented in Figure 2, right.

The system of equations (18) imposes the intersection of the ellipse $\cos \hat{\phi}_{1}\left(\theta_{3}\right)$ with the family of ellipses $\cos \hat{\phi}_{2}\left(\theta_{1}, \theta_{5}\right)$. Our purpose is the determination of the intervals of values of the input angles, $\theta_{1}, \theta_{3}$, or $\theta_{5}$, for which such intersection exists, which obviously correspond to the feasible values of these variables for the mechanism to close.

\subsection{Bounding values of $\theta_{5}$ and $\theta_{1}$}

Here, we show the process to find the bounding values of the feasible intervals of $\theta_{5}$. The process to find the bounding values for $\theta_{1}$ is completely analogous, simply interchanging the roles of $\theta_{1}$ and $\theta_{5}$.

For a fixed value of $\theta_{5}$, the possible values that $\cos \hat{\phi}_{2}\left(\theta_{1}, \theta_{5}\right)$ can take when varying $\theta_{1}$ consist in a single ellipse, which may intersect the ellipse $\cos \hat{\phi}_{1}\left(\theta_{3}\right)$ at a maximum of four points. Since the ellipses $\cos \hat{\phi}_{2}\left(\theta_{1}, \theta_{5}\right)$ change 

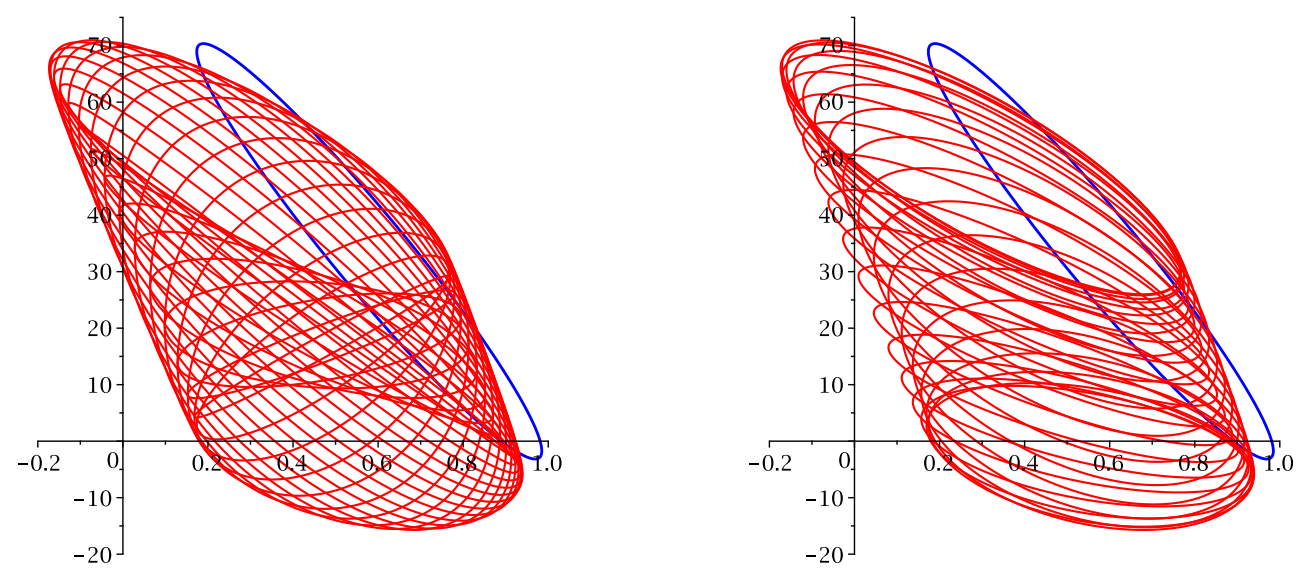

Figure 2: Ellipse $\cos \hat{\phi}_{1}\left(\theta_{3}\right)$ (blue) and family of ellipses $\cos \hat{\phi}_{2}\left(\theta_{1}, \theta_{5}\right)$ (red). Left: Using $\theta_{5}$ as parameter. Right: Using $\theta_{1}$ as parameter.

smoothly with $\theta_{5}$, a necessary condition for a feasible value of $\theta_{5}$ to be a bounding value is that the corresponding ellipse becomes tangent to the ellipse $\cos \hat{\phi}_{1}\left(\theta_{3}\right)$. We can distinguish two cases: that the two ellipses are non-intersecting and tangent at one point, or that they are tangent at one point and intersecting at two other points ${ }^{1}$. The first situation is the only one that can give raise to a transition between contact and non-contact of the ellipses, as corresponds to a bounding value of $\theta_{5}$. The second situation can only give rise to a change in the number of intersection points, namely, between 2 and 4, and this corresponds to a value of $\theta_{5}$ bounding just one of the two possible modes of assembly.

An intersection point of the ellipses corresponds to a real root of the quartic for $T_{1}=\tan \left(\theta_{1} / 2\right)$ obtained in Section 5.1 , while a tangency point corresponds to a double real root of this quartic. It is a well established result that the nature of the roots of a quartic of the form $a x^{4}+b x^{3}+c x^{2}+d x+e$ can be assessed by analyzing the signs of the polynomials of its coefficients defined next:

$$
\begin{aligned}
\Delta= & 256 a^{3} e^{3}-192 a^{2} b d e^{2}-128 a^{2} c^{2} e^{2}+144 a^{2} c d^{2} e-27 a^{2} d^{4}+144 a b^{2} c e^{2}-6 a b^{2} d^{2} e-80 a b c^{2} d e+ \\
& 18 a b c d^{3}+16 a c^{4} e-4 a c^{3} d^{2}-27 b^{4} e^{2}+18 b^{3} c d e-4 b^{3} d^{3}-4 b^{2} c^{3} e+b^{2} c^{2} d^{2} \\
\mathrm{D}= & 64 a^{3} e-16 a^{2} c^{2}+16 a b^{2} c-16 a^{2} b d-3 b^{4} \\
\mathrm{P}= & 8 a c-3 b^{2} \\
\mathrm{R}= & b^{3}+8 d a^{2}-4 a b c
\end{aligned}
$$

The necessary and sufficient condition for the quartic to have a double root (real or complex) is $\Delta=0$. If in addition the following condition is fulfilled:

$$
(\mathrm{D}>0) \vee(\mathrm{P}>0 \wedge(\mathrm{D} \neq 0 \vee \mathrm{R} \neq 0))
$$

then the quartic has just one double real root and two complex roots, which is just the condition to have a bound of the input variable. Thus, our first step will be to find the values of $\theta_{5}$ for which $\Delta=0$ and then check each of them to see if condition (19) is satisfied. Note that the expression of $\Delta$ is a 6th degree polynomial of the coefficients, which in our case are 2nd degree polynomials in $\sin \theta_{5}$ and $\cos \theta_{5}$, as explained in Section 5.1. If we perform the substitution $T_{5}=\tan \left(\theta_{5} / 2\right)$ to solve the equation, we end up with a polynomial of degree 24 for $T_{5}$, whose real roots can be readily computed with a mathematical software such as Maple.

Figure 3, left, shows, for the example case, the four ellipses $\cos \hat{\phi}_{2}\left(\theta_{1}, \theta_{5}\right)$ tangent to the ellipse $\cos \hat{\phi}_{1}\left(\theta_{3}\right)$ corresponding to the four real solutions found for $\theta_{5}$ satisfying $\Delta=0$. The red ellipses are those for which condition (19) is fulfilled and correspond to the global bounds of $\theta_{5}$. The green ellipses are those for which condition (19) is not fulfilled and correspond to the values of the input angle $\theta_{5}$ bounding just one of the two possible modes of assembly of the mechanism. Similarly, Figure 3, right, shows the ellipses $\cos \hat{\phi}_{2}\left(\theta_{1}, \theta_{5}\right)$ corresponding to the four real solutions

\footnotetext{
${ }^{1}$ Unusual singular situations may also occur, including tangency at two different points, and others. Since such situations do not occur for generic link dimensions, they are not further analyzed here.
} 
found when the same process is performed for $\theta_{1}$. Table 2 gives the numerical values of the solutions obtained for both variables.
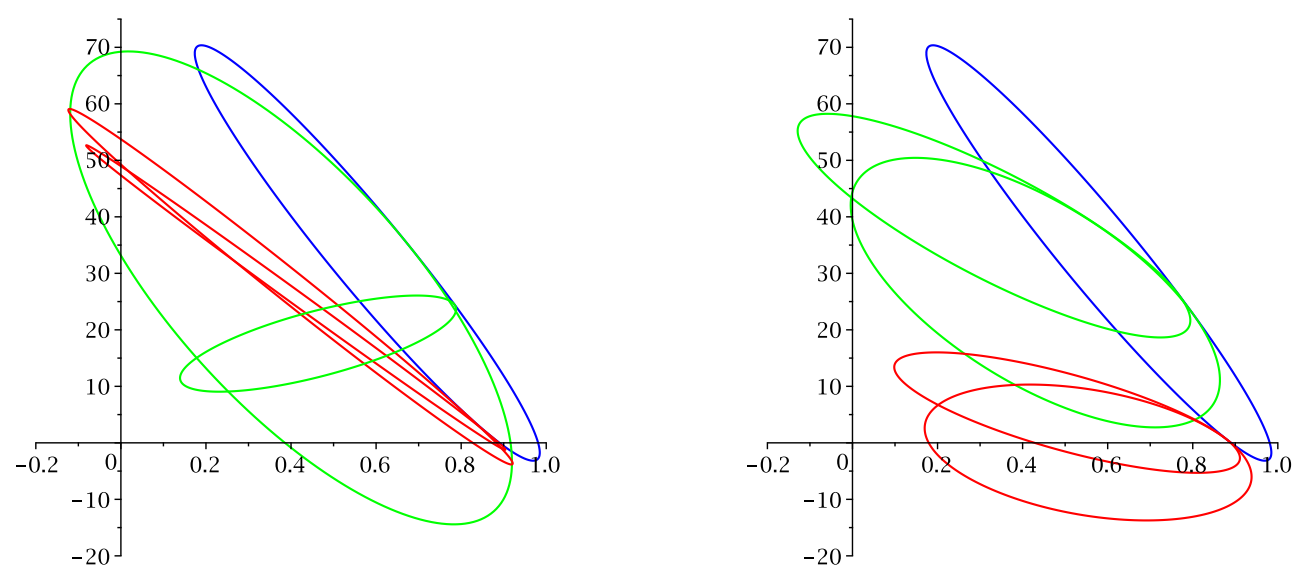

Figure 3: Ellipses of the family $\cos \hat{\phi}_{2}\left(\theta_{1}, \theta_{5}\right)$ tangent to $\cos \hat{\phi}_{1}\left(\theta_{3}\right)$. Left: Using $\theta_{5}$ as parameter. Right: Using $\theta_{1}$ as parameter.

Table 2: Values of variables $\theta_{5}$ and $\theta_{1}$ for which the ellipses are tangent.

\begin{tabular}{|c|c|c|}
\cline { 2 - 3 } \multicolumn{1}{c|}{} & $\theta_{5}$ & $\theta_{1}$ \\
\hline$\Delta=0$ AND & $50.47198^{\circ}$ & $126.86437^{\circ}$ \\
Condition (19) $=$ True & $69.35084^{\circ}$ & $168.41778^{\circ}$ \\
\hline$\Delta=0$ AND & $148.78672^{\circ}$ & $43.97517^{\circ}$ \\
Condition (19) $=$ False & $307.29956^{\circ}$ & $268.49318^{\circ}$ \\
\hline
\end{tabular}

\subsection{Bounding values of $\theta_{3}$}

To find the bounds of $\theta_{3}$ we have to follow a different approach. In this case we have to consider the intersection of the ellipse $\cos \hat{\phi}_{1}\left(\theta_{3}\right)$ with the whole family of ellipses $\cos \hat{\phi}_{2}\left(\theta_{1}, \theta_{5}\right)$. The bounding values of $\theta_{3}$ are those corresponding to the points of the ellipse $\cos \hat{\phi}_{1}\left(\theta_{3}\right)$ lying at the boundary of the region filled by the ellipses $\cos \hat{\phi}_{2}\left(\theta_{1}, \theta_{5}\right)$ (see Fig. $2)$, that is, the intersection of $\cos \hat{\phi}_{1}\left(\theta_{3}\right)$ with the envelope of the ellipses $\cos \hat{\phi}_{2}\left(\theta_{1}, \theta_{5}\right)$.

The envelope curve of a one-parameter family of curves $f(x, y, \theta)=0$, where $\theta$ is the parameter, is commonly identified with the discriminant curve [19], defined as the set of points satisfying:

$$
\left\{\begin{array}{l}
f(x, y, \theta)=0 \\
\frac{\partial f(x, y, \theta)}{\partial \theta}=0,
\end{array}\right.
$$

but, since this may include points in the interior of the region filled by the curves, some authors consider as the envelope the subset of the discriminant curve lying at the boundary of the filled region [20].

In our case, for a given value of the parameter $\theta_{5}$, the system (17) provides the parametric equation of an ellipse with parameter $\theta_{1}$, and our first step is converting it into a single equation by eliminating $\theta_{1}$. We rewrite (17) by renaming $\cos \phi_{2}=x$ and $d_{2} \sin \phi_{2}=y$ :

$$
\left\{\begin{array}{l}
x=F+G \cos \theta_{1}+H \sin \theta_{1} \\
y=J+K \cos \theta_{1}+L \sin \theta_{1}
\end{array}\right.
$$

After eliminating $\theta_{1}$, we get the equation of the parametric family of ellipses $\cos \hat{\phi}_{2}\left(\theta_{1}, \theta_{5}\right)$ in the plane $(x, y)$ :

$$
f\left(x, y, \theta_{5}\right)=(H x-G y-K F+G J)^{2}+(L x-H y-L F+H J)^{2}-(L G-H K)^{2}=0,
$$


where the coefficients are functions of $\theta_{5}$, as noted in Section 4. Writing $f^{\prime}\left(x, y, \theta_{5}\right)=\partial f\left(x, y, \theta_{5}\right) / \partial \theta_{5}$, and applying the substitution $T_{5}=\tan \left(\theta_{5} / 2\right)$ in both $f\left(x, y, \theta_{5}\right)$ and $f^{\prime}\left(x, y, \theta_{5}\right)$, we get $g\left(x, y, T_{5}\right)$ and $g^{\prime}\left(x, y, T_{5}\right)$, respectively, so that the discriminant curve of $\cos \hat{\phi}_{2}\left(\theta_{1}, \theta_{5}\right)$ is given by:

$$
\left\{\begin{array}{l}
g\left(x, y, T_{5}\right)=0 \\
g^{\prime}\left(x, y, T_{5}\right)=0
\end{array}\right.
$$

To find the intersection of the discriminant curve with the ellipse $\cos \hat{\phi}_{1}\left(\theta_{3}\right)$, we first transform the parametric expression of the ellipse given by (16) into a single equation through the elimination of $\theta_{3}$. We do this by isolating sin $\theta_{3}$ and $\cos \theta_{3}$ and using the identity $\sin ^{2} \theta_{3}+\cos ^{2} \theta_{3}=1$ to get:

$$
\mathcal{E}(x, y)=(E x-E A)^{2}+(D x-B y-D A+B C)^{2}-(B E)^{2}=0 .
$$

where $x=\cos \phi_{1}$ and $y=d_{1} \sin \phi_{1}$. Finally, we can form the system:

$$
\left\{\begin{array}{l}
g\left(x, y, T_{5}\right)=0 \\
g^{\prime}\left(x, y, T_{5}\right)=0 \\
\mathcal{E}(x, y)=0
\end{array}\right.
$$

Solving this system, we get the points $(x, y)$ of the intersection and, since $x=\cos \phi_{1}$ and $y=d_{1} \sin \phi_{1}$, we can obtain $\theta_{3}$ using (16). Alternatively, the intersection points can be obtained by taking $\theta_{1}$, instead of $\theta_{5}$, as the parameter of $\cos \hat{\phi}_{2}\left(\theta_{1}, \theta_{5}\right)$.

Figure 4 shows, for our case example, the two discriminant curves of the family of ellipses defined by (22) obtained by taking $\theta_{5}$ and $\theta_{1}$, respectively, as parameter of $\cos \hat{\phi}_{2}\left(\theta_{1}, \theta_{5}\right)$. The ellipse $\cos \hat{\phi}_{1}$ defined by (23) is represented in blue and the intersection points are marked in red. Observe that the two discriminant curves differ in a segment that appears when the parameter is $\theta_{5}$ but not when the parameter is $\theta_{1}$. This corresponds to a value of $\theta_{5}$ for which the ellipse in the family degenerates into this segment, a fact that does not happen when the parameter is $\theta_{1}$. Solving the
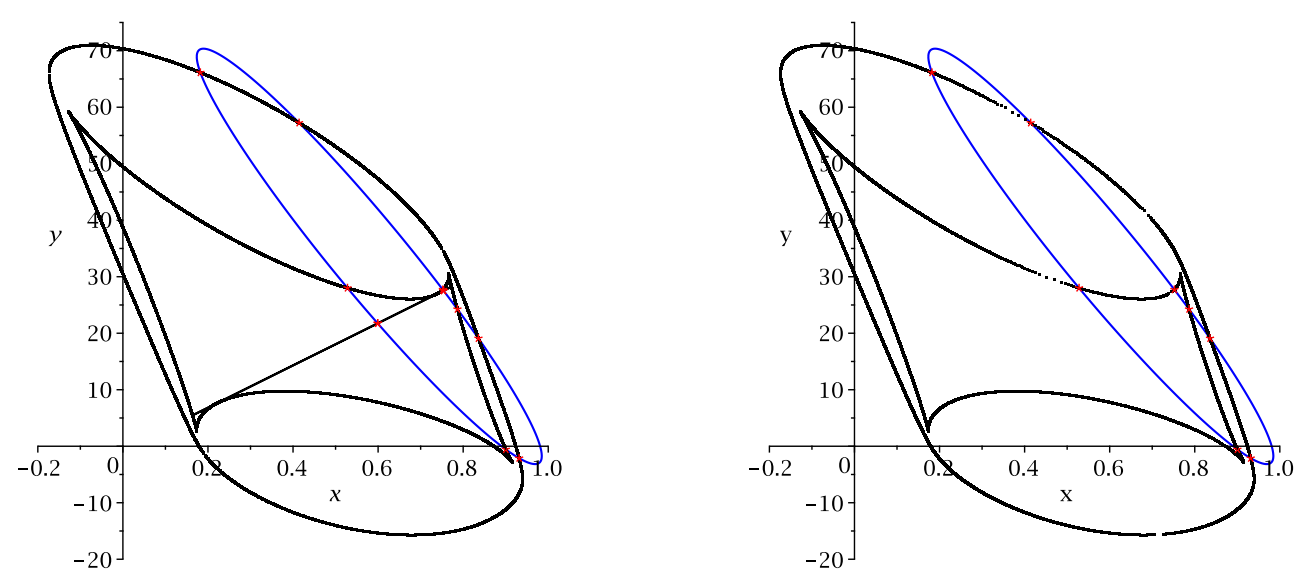

Figure 4: Intersection of the ellipse $\cos \hat{\phi}_{1}\left(\theta_{3}\right)$ with the discriminant curve of the family of ellipses $\cos \hat{\phi}_{2}\left(\theta_{1}, \theta_{5}\right)$. Left: $\cos \hat{\phi}_{2}\left(\theta_{1}, \theta_{5}\right)$ parameterized by $\theta_{5}$. Right: $\cos \hat{\phi}_{2}\left(\theta_{1}, \theta_{5}\right)$ parameterized by $\theta_{1}$

system (24), we obtain 48 solutions, among which 12 are real when the parameter of $\cos \hat{\phi}_{2}\left(\theta_{1}, \theta_{5}\right)$ is $\theta_{5}$, and only 8 when the parameter is $\theta_{1}$ (see Table 3 ). The four additional real solutions of the first case are in fact just two double roots, and correspond to the intersections of the ellipse $\cos \hat{\phi}_{1}$ with the additional segment of the discriminant curve with parameter $\theta_{5}$. Each one of these solutions corresponds to a value of $\theta_{5}$ which accidentally coincides for two different configurations with a same value of $\theta_{3}$. This kind of coincidence, however, is not relevant in any way and does not have any implication on the mobility of the mechanism. Figure 5 shows the linkage with the parameters given in Table 2 in the configuration corresponding to the extreme position of $\theta_{3}=293.99367^{\circ}$. 
Table 3: Values of variable $\theta_{3}$ for which the ellipse $\cos \hat{\phi}_{1}\left(\theta_{3}\right)$ intersects the discriminant curve of $\cos \hat{\phi}_{2}\left(\theta_{1}, \theta_{5}\right)$.

\begin{tabular}{|c|c|}
\hline parameter $=\theta_{5}$ & parameter $=\theta_{1}$ \\
\hline $11.76345^{\circ}$ & $11.76345^{\circ}$ \\
$82.74850^{\circ}$ & $82.74850^{\circ}$ \\
$92.79834^{\circ}$ & \\
$142.32368^{\circ}$ & $142.32368^{\circ}$ \\
$150.31604^{\circ}$ & $150.31604^{\circ}$ \\
$230.73735^{\circ}$ & $230.73735^{\circ}$ \\
$239.25964^{\circ}$ & $239.25964^{\circ}$ \\
$244.49660^{\circ}$ & \\
$244.75767^{\circ}$ & $244.75767^{\circ}$ \\
$293.99367^{\circ}$ & $293.99367^{\circ}$ \\
\hline
\end{tabular}

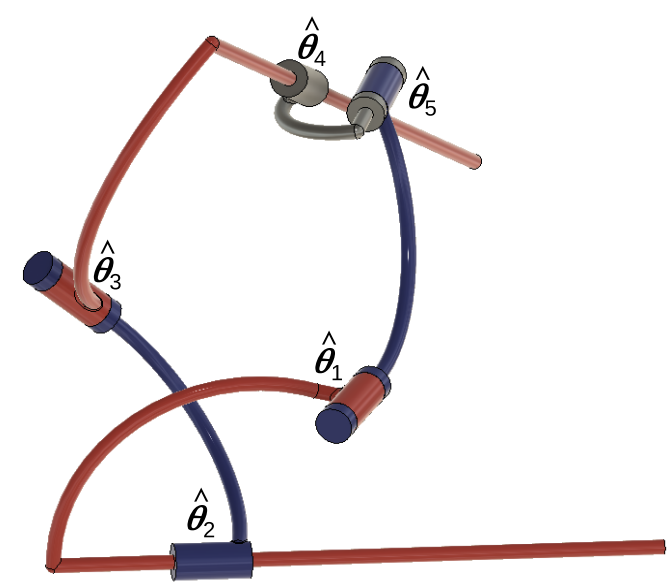

Figure 5: The example RCRCR mechanism in the extreme position of $\theta_{3}=293.99367^{\circ}$.

\section{Determination of the feasible intervals for the input variables}

The bounding values obtained in Section 6 partition the domain of each variable into circular intervals which are either feasible or unfeasible for that variable. It only remains to determine which of these intervals actually contain the solution values. A direct way to determine if one of the intervals for variable $\theta_{i}$ is feasible or not consists in selecting an interior point of the interval and check if it gives rise to some real solution using whichever of the two input-output functions obtained in Section 6 having $\theta_{i}$ as the input variable. Next, we apply this procedure to our example.

\subsection{Intervals for $\theta_{5}$ and $\theta_{1}$}

For variable $\theta_{5}$ we can use the input-output function $\theta_{1}\left(\theta_{5}\right)$, which is simpler than the alternative $\theta_{3}\left(\theta_{5}\right)$. Since we have only two bounding values (see Table 2 ), there are two circular intervals to consider: $\left[50.47198^{\circ}, 69.35083^{\circ}\right]$ and $\left[69.35083^{\circ}, 50.47198^{\circ}+360^{\circ}\right]$. We check a value in the first interval and see that it gives no solution, so we conclude that the feasible interval must be $\theta_{5} \in\left[69.35083^{\circ}, 50.47198^{\circ}+360^{\circ}\right]$. In a similar way, for variable $\theta_{1}$ we use $\theta_{5}\left(\theta_{1}\right)$ to determine that the solution interval is $\theta_{1} \in\left[168.41778^{\circ}, 126.86437^{\circ}+360^{\circ}\right]$. Thus, a single test for each variable is enough to determine the right intervals. Additionally, by considering the values in Table 2 not satisfying condition (19), we can also obtain the intervals allowed for each mode of assembly. We just need to check an interior point in each resulting interval to see if there are two or four solutions. 
Figure 6 shows, at top, the input-output functions $\theta_{1}\left(\theta_{5}\right)$ and $\theta_{3}\left(\theta_{5}\right)$ for the input variable $\theta_{5}$ with its feasible interval marked in bold on the horizontal axis. Vertical lines are placed on each extreme value obtained for $\theta_{5}$, showing their coincidence with the return points of this joint. Red lines correspond to global bounds of the solution interval, while green lines correspond to bounds of just one mode of assembly. Similarly, Figure 6, bottom, shows the input-output functions $\theta_{5}\left(\theta_{1}\right)$ and $\theta_{3}\left(\theta_{1}\right)$ for the input variable $\theta_{1}$ and its feasible intervals.
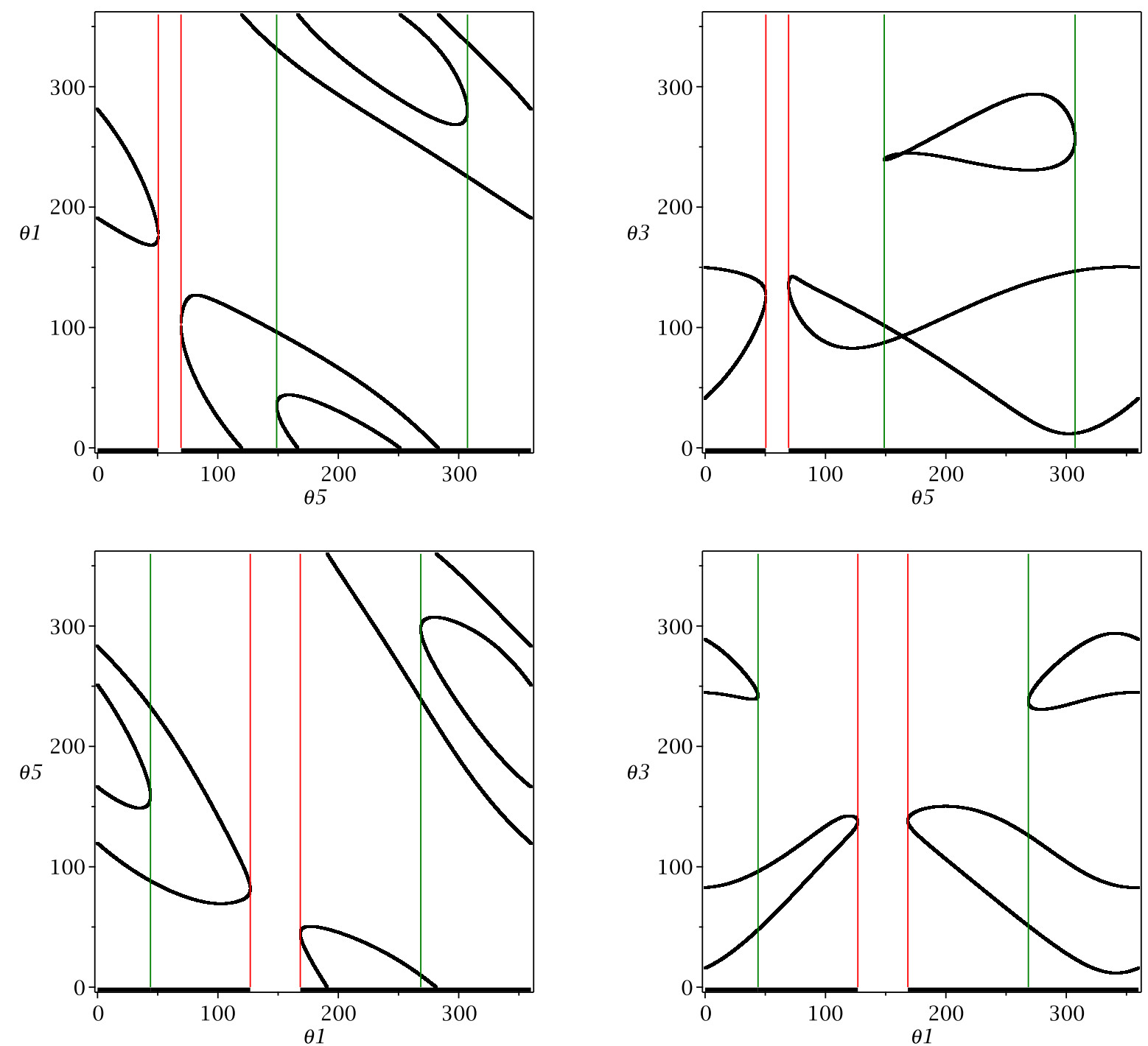

Figure 6: Solution intervals for the input variables $\theta_{5}$ (top) and $\theta_{1}$ (bottom)

\subsection{Intervals for $\theta_{3}$}

In the case of $\theta_{3}$ we found that the ellipse for $\cos \hat{\phi}_{1}\left(\theta_{3}\right)$ intersects the discriminant curve of the family of ellipses for $\cos \hat{\phi}_{2}\left(\theta_{1}, \theta_{5}\right)$ at 10 points when the parameter of the family is $\theta_{5}$, and 8 when the parameter is $\theta_{1}$. In this case, it is harder to tell which of them lay on the envelope, a necessary condition to correspond to a global bound, and we rely on the method of checking one point into each resulting interval. Since the envelope must be contained in both discriminant curves, we can ignore the two extra values obtained when $\theta_{5}$ is the parameter of the family and just check an interior point in each one of the 8 circular intervals determined by the 8 values common to both cases. Doing this and joining consecutive feasible intervals we get two solution intervals for $\theta_{3}:\left[11.76345^{\circ}, 150.31604^{\circ}\right.$ ] and $\left[230.73735^{\circ}, 293.99367^{\circ}\right]$, one for each assembly mode. The result is shown in Figure 7, top. Note that all return points of the joint are correctly detected. 

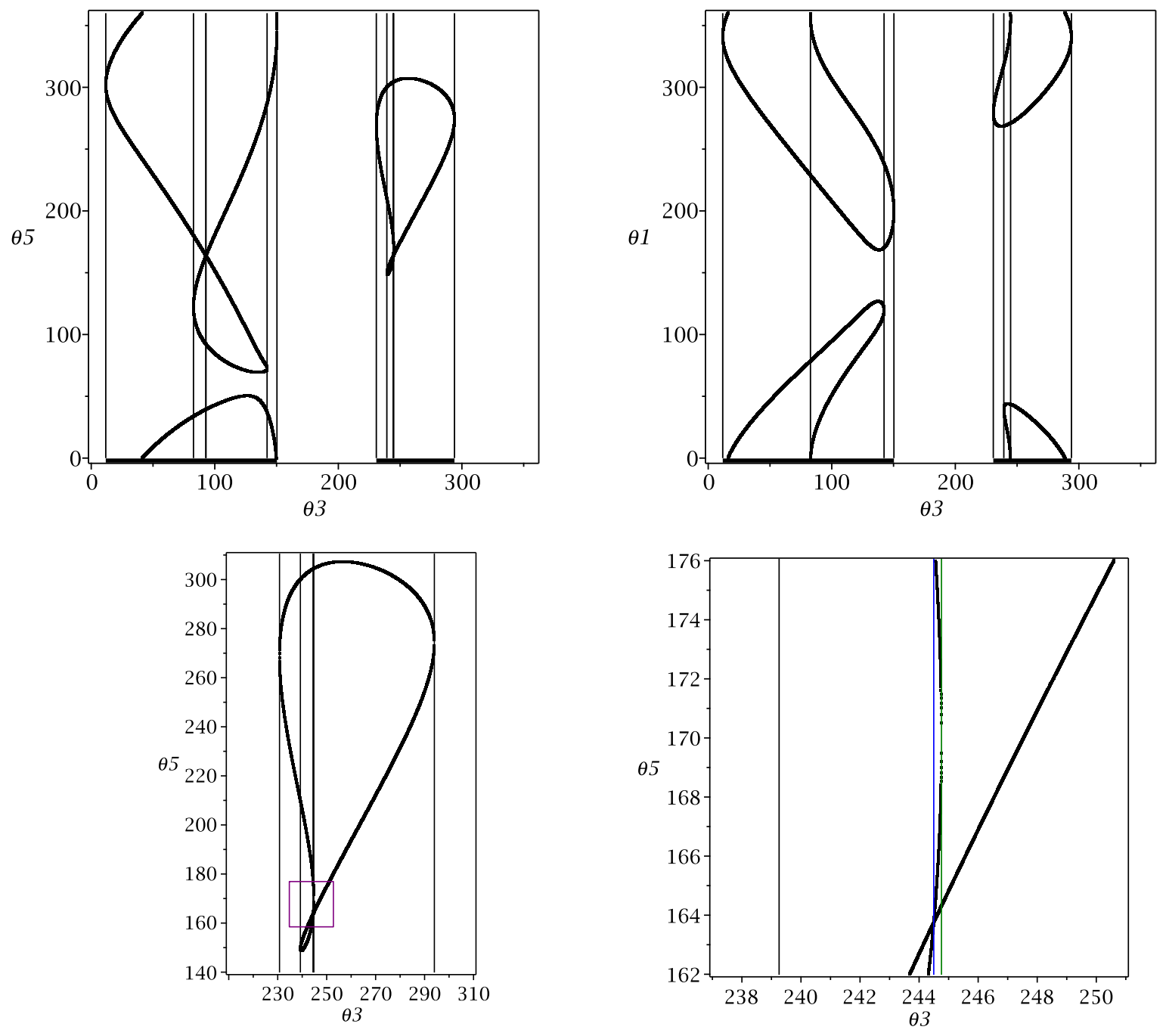

Figure 7: Top: Ranges for the input variable $\theta_{3}$. Bottom: detail of the second assembly mode.

In the top left graph of Figure 7, representing $\theta_{5}\left(\theta_{3}\right)$, there are also represented the two extra values obtained for the intersection with the discriminant curve, and it can be seen that they coincide with the values of $\theta_{3}$ where the curve intersects with itself reducing the number of solutions from 4 to 3 . This is clearly seen on the assembly mode appearing at left on the graph, but not on that at right due to the proximity of this value with that of a return point of the variable. The bottom of the figure shows, at left, a first amplification of this mode of assembly where a small squared area is marked around the intersection, and, at right, a further amplification of the marked area where the two lines appear visibly separated and coinciding with the crossing and the return points, respectively.

Table 4 summarizes the results obtained for ranges and return points of the input variables in each assembly mode.

\section{Conclusions}

We have presented a method to obtain analytical expressions for the extrema of the solution intervals of the input variables corresponding to the R pairs of a spatial RCRCR mechanism. Solution intervals are determined for each one of the two possible assembly modes of the mechanism. Return points of the input variable which are not interval limits are also identified as local extrema, which correspond to singular configurations of the mechanism, relevant in many aspects of mechanism analysis and synthesis. 
Table 4: Intervals and return points of the input variables.

\begin{tabular}{|c|c|c|c|c|}
\cline { 2 - 5 } \multicolumn{1}{c|}{} & \multicolumn{2}{c|}{ Assembly mode 1 } & \multicolumn{2}{c|}{ Assembly mode 2 } \\
\cline { 2 - 5 } \multicolumn{1}{c|}{ feasible range } & other return points & feasible range & other return points \\
\hline$\theta_{1}$ & {$\left[168.41778^{\circ}, 126.86437^{\circ}+360^{\circ}\right]$} & & {$\left[268.49318^{\circ}, 43.97517^{\circ}+360^{\circ}\right]$} & \\
$\theta_{5}$ & {$\left[69.35083^{\circ}, 50.47198^{\circ}+360^{\circ}\right]$} & & {$\left[148.78672^{\circ}, 307.29956^{\circ}\right]$} & \\
$\theta_{3}$ & {$\left[11.76345^{\circ}, 150.31604^{\circ}\right]$} & $82.74850^{\circ}, 142.32368^{\circ}$ & {$\left[230.73735^{\circ}, 293.99367^{\circ}\right]$} & $239.25964^{\circ}, 244.75767^{\circ}$ \\
\hline
\end{tabular}

Future extensions of this work can be addressed to obtain the solution intervals for the non-input variables, i.e., those corresponding to the $\mathrm{C}$ pairs of the mechanism, and also to deal with other five-link spatial linkages.

\section{Acknowledgements}

This work is supported by the Spanish Ministry of Economy and Competitiveness through the I+D projects DPI2014-57220-C2-2-P and DPI2017-88282-P.

\section{References}

[1] S. Bai, Coupler-link mobility analysis of planar four-bar linkages, in: P. Wenger, P. Flores (Eds.), New Trends in Mechanism and Machine Science, Springer International Publishing, 2017, pp. 41-49.

[2] E. Celaya, C. Torras, On finding the set of inverse kinematic solutions for redundant manipulators, in: J. Angeles, G. Hommel, P. Kovács (Eds.), Computational Kinematics, Kluwer Academic Publishers, 1993, pp. 85-94.

[3] E. Celaya, C. Torras, Solving multiloop linkages with limited-range joints, Mechanism and Machine Theory 29 (3) (1994) $373-391$.

[4] E. Celaya, Interval propagation for solving parallel spherical mechanisms, in: J. Lenarčič, F. Thomas (Eds.), Advances in Robot Kinematics, 2002, pp. 415-422.

[5] E. Celaya, T. Creemers, L. Ros, Exact interval propagation for the efficient solution of position analysis problems on planar linkages, Mechanism and Machine Theory 54 (2012) 116-131.

[6] A. T. Yang, Displacement analysis of spatial five-link mechanisms using (3x3) matrices with dual-number elements, ASME Journal of Engineering for Industry 91 (1) (1969) 152-157.

[7] H. H. Cheng, S. Thompson, Computer-aided displacement analysis of spatial mechanisms using the $\mathrm{C}^{H}$ programming language, Advances in Engineering Software (23) (1995) 163-172.

[8] T. Creemers, J. M. Porta, L. Ros, F. Thomas, Fast multiresolutive approximations of planar linkage configuration spaces, in: Proc. of the IEEE Int. Conf. on Robotics and Automation (ICRA), 2006, pp. 1511-1517.

[9] J. M. Porta, L. Ros, F. Thomas, A linear relaxation technique for the position analysis of multi-loop linkages, IEEE Transactions on Robotics 25 (2) (2009) 225-239.

[10] J.-P. Merlet, Interval analysis for certified numerical solution of problems in robotics, International Journal of Applied Mathematics and Computer Science 19 (3) (2009) 399-412.

[11] K. H. Hunt, Kinematic Geometry of Mechanisms, Oxford University Press., Oxford, 1978.

[12] J. E. Baker, K. J. Wampler, Limit positions of spatial linkages via screw system theory, in: Asme Design Engineering Technical Conference. Paper no. 74-DET-107, New York, 1974

[13] J. E. Baker, On the investigation of extrema in linkage analysis, using screw system algebra, Mechanism and Machine Theory 13 (1978) $333-343$.

[14] R. L. Williams, C. F. Reinholtz, Mechanism link rotatability and limit position analysis using polynomial discriminants, ASME Journal of Mechanisms, Transmissions, and Automation in Design 109(2) (1987) 178-182.

[15] C. Borcea, I. Streinu, Extremal configurations of manipulators with revolute joints, in: ASME/IFToMM International Conference on Reconfigurable Mechanisms and Robots, 2009, pp. 342-347.

[16] G. R. Pennock, A. T. Yang, Application of dual-number matrices to the inverse kinematics problem of robot manipulators, Journal of Mechanisms, Transmissions, and Automation in Design 107 (2) (1985) 201-208.

[17] J. Duffy, H. Y. Habib-Olahi, A displacement analysis of spatial five-link 3R-2C mechanisms-I. on the closures of the rcrcr mechanism, Journal of Mechanisms 6 (3) (1971) 289-301.

[18] J. Duffy, Analysis of Mechanisms and Robot Manipulators, Edward Arnold (Publishers) Ltd., London, 1980.

[19] J. W. Bruce, P. J. Giblin, Curves and Singularities, Cambridge University Press, 1984.

[20] J. W. Rutter, Geometry of Curves, CRC Press, 2000. 